\title{
Unified description of inelastic propensity rules for electron transport through nanoscale junctions
}

Paulsson, Magnus; Frederiksen, Thomas; Ueba, Hiromu; Lorente, Nicolas; Brandbyge, Mads

Published in:

Physical Review Letters

Link to article, DOI:

10.1103/PhysRevLett.100.226604

Publication date:

2008

Document Version

Publisher's PDF, also known as Version of record

Link back to DTU Orbit

Citation (APA):

Paulsson, M., Frederiksen, T., Ueba, H., Lorente, N., \& Brandbyge, M. (2008). Unified description of inelastic propensity rules for electron transport through nanoscale junctions. Physical Review Letters, 100(22), 226604. https://doi.org/10.1103/PhysRevLett.100.226604

\section{General rights}

Copyright and moral rights for the publications made accessible in the public portal are retained by the authors and/or other copyright owners and it is a condition of accessing publications that users recognise and abide by the legal requirements associated with these rights.

- Users may download and print one copy of any publication from the public portal for the purpose of private study or research.

- You may not further distribute the material or use it for any profit-making activity or commercial gain

- You may freely distribute the URL identifying the publication in the public portal 


\title{
Unified Description of Inelastic Propensity Rules for Electron Transport through Nanoscale Junctions
}

\author{
Magnus Paulsson, ${ }^{1,2, *}$ Thomas Frederiksen, ${ }^{3,4}$ Hiromu Ueba, ${ }^{2}$ Nicolás Lorente, ${ }^{5,2}$ and Mads Brandbyge ${ }^{6}$ \\ ${ }^{1}$ Division of Physics, School of Pure and Applied Natural Science, University of Kalmar, 39182 Kalmar, Sweden \\ ${ }^{2}$ Department of Electronics, Toyama University, Gofuku, Toyama, 930-8555, Japan \\ ${ }^{3}$ Donostia International Physics Center (DIPC), Manuel de Landizabal Pasealekua 4, 20018 Donostia, Spain \\ ${ }^{4}$ CIC nanoGUNE Consolider, Mikeletegi Pasealekua 56, 20009 Donostia, Spain \\ ${ }^{5}$ Centro de Investigación en Nanociencia y Nanotecnología, CSIC-ICN, Campus de la Universitat Autònoma de Barcelona, \\ 08193 Bellaterra, Spain \\ ${ }^{6}$ DTU Nanotech, NanoDTU, Technical University of Denmark, 2800 Lyngby, Denmark
}

(Received 21 November 2007; published 5 June 2008)

\begin{abstract}
We present a method to analyze the results of first-principles based calculations of electronic currents including inelastic electron-phonon effects. This method allows us to determine the electronic and vibrational symmetries in play, and hence to obtain the so-called propensity rules for the studied systems. We show that only a few scattering states - namely those belonging to the most transmitting eigenchannels - need to be considered for a complete description of the electron transport. We apply the method on first-principles calculations of four different systems and obtain the propensity rules in each case.
\end{abstract}

PACS numbers: $72.10 .-\mathrm{d}, 73.23 .-\mathrm{b}$

Electronic transport through atomic-size junctions is of immense scientific and technological interest. The importance of inelastic effects in electronic currents has been revealed in several ground-breaking experiments leading to the detection and identification of single molecules [1], chemical reactions $[2,3]$, the detection of vibrations in atomic wires [4], the detection of inelastic effects by fluorescence [5], the modification of electron transport in nanotubes [6], the molecular motion induced by electronic currents [7], and the hydrogen detection in atomic wires [8], just to cite a few examples. Of particular importance due to its spreading use is the case of vibrational spectroscopy where the conductance changes due to phonon emission is measured [9-11]. This is often referred to as point contact spectroscopy or inelastic electron tunneling spectroscopy (IETS) [1]. However, experiments alone are not able to give direct insight into the fundamental question on how the detailed atomic structure correlates with the electrical transport properties. There is experimental evidence of approximate selection rules (propensity rules [12]) such that only a small number out of the many possible vibrational modes give an inelastic signal. These propensity rules yield clues to the geometric and electronic structure of the junctions. It is therefore of fundamental interest to compare the experimental results with first-principles calculations.

Existing calculations of inelastic effects in electron transport have been developed either for particular cases [12-14] or for simplified (one-level) models [15,16]. Firstprinciples methods capable of treating both weak and strong coupling to the electrodes have also been developed [17-19]. However, the results of such detailed calculations involve many electronic states and vibrational modes. An advanced analysis is therefore needed in order to provide insight into the propensity rules.
In this Letter we propose a method for analysis of the inelastic transport based on just a few selected electronic scattering states, namely, those belonging to the most transmitting eigenchannels at the Fermi energy $\left(\varepsilon_{\mathrm{F}}\right)$ [20]. These scattering states typically have the largest amplitude inside the junction and thus account for the majority of the electron-phonon (e-ph) scattering. To illustrate our method of analysis and to develop an understanding of the propensity rules we consider four cases: (i) atomic gold-wires, and (ii) molecular junctions, as well as scanning tunneling microscope (STM) setups in the (iii) resonant, and (iv) nonresonant limits. The propensity rules can in these cases be understood from $e$-ph induced transitions between scattering states of a few eigenchannels.

Inelastic scattering of electrons in a device under bias can be modeled using nonequilibrium Green's functions (NEGF) $[15,16,19]$. In particular, the lowest order expansion (LOE) of the NEGF equations provides a tractable description of phonon scattering in first-principles calculations [17]. This approximation assumes a weak $e$-ph coupling (M) and that the electronic structure changes slowly over a phonon energy $(\hbar \omega)$. It is therefore not applicable to strong $e$-ph coupling. In the zero-temperature limit, the conductance is

$$
G^{\mathrm{LOE}}=G_{0} \tau+\sum_{\lambda} e \gamma_{\lambda}^{\mathrm{LOE}} \theta\left(|e V|-\hbar \omega_{\lambda}\right)+G_{\lambda}^{\mathrm{Asym}},
$$

which can be divided into the Landauer term, with the transmission $\tau$ (at $\varepsilon_{\mathrm{F}}$ ) times the conductance quantum $G_{0}$, and inelastic corrections in the conductance from each vibrational mode. In this formulation we have separated the inelastic contribution in a symmetric term, with respect to bias, from phonon absorption and emission processes, and an asymmetric term $G_{\lambda}^{\text {Asym }} . G_{\lambda}^{\text {Asym }}$ is small in the cases studied here since it is (i) strictly zero for symmetric 
junctions and (ii) negligible in the STM configuration when very close to or very far from resonance [17,21]. For these reasons we ignore $G_{\lambda}^{\mathrm{Asym}}$ in the following discussion. Each mode thus gives a step $\theta\left(|e V|-\hbar \omega_{\lambda}\right)$ in the conductance at the phonon energy. The magnitude and sign of this step is given by the scattering rate per excess bias

$$
\begin{aligned}
\gamma_{\lambda}^{\mathrm{LOE}}= & \frac{e}{\pi \hbar} \operatorname{Tr}\left[\mathbf { G } ^ { \dagger } \boldsymbol { \Gamma } _ { L } \mathbf { G } \left\{\mathbf{M}_{\lambda} \mathbf{G} \boldsymbol{\Gamma}_{R} \mathbf{G}^{\dagger} \mathbf{M}_{\lambda}\right.\right. \\
& \left.\left.+\frac{i}{2}\left(\boldsymbol{\Gamma}_{R} \mathbf{G}^{\dagger} \mathbf{M}_{\lambda} \mathbf{A} \mathbf{M}_{\lambda}-\text { H.c. }\right)\right\}\right],
\end{aligned}
$$

where $\mathbf{G}$ is the retarded Green's function at $\varepsilon_{\mathrm{F}}, \mathbf{A}=i(\mathbf{G}-$ $\left.\mathbf{G}^{\dagger}\right)$ the spectral function, and $\boldsymbol{\Gamma}_{L, R}$ the couplings to the leads.

It is instructive to use a one-level model to get an understanding of the sign of the conductance step. The one-level model couples a single electronic level to two electronic leads and a localized vibration [17,21]. A phase diagram is plotted in Fig. 1 for the ratio of the coupling to the two leads $\alpha=\Gamma_{R} / \Gamma_{L}$ and the transmission $\tau$ at $\varepsilon_{\mathrm{F}}$. For this model the maximal transmission is $\tau_{\max }=4 \alpha /(1+\alpha)^{2}$ corresponding to the on-resonance case. The crossover from a decrease to an increase in the conductance is given by the $1 / 2$ rule $[17,22,23]$, i.e., at $\tau_{\text {crossover }}=\tau_{\max } / 2$. Backscattering dominates the high-transmission system leading to a decrease of the conductance while forward scattering leads to an increase in the low-transmitting case [24].

The scattering rate $\gamma^{\mathrm{LOE}}$ may be interpreted as a competition between an inelastic process, the first term of Eq. (2), that increases the current and an elastic correction, the second term of Eq. (2), that decreases the current $[13,15,24]$. In the case of low transmission, where only the inelastic term needs to be considered, Troisi et al. [12]

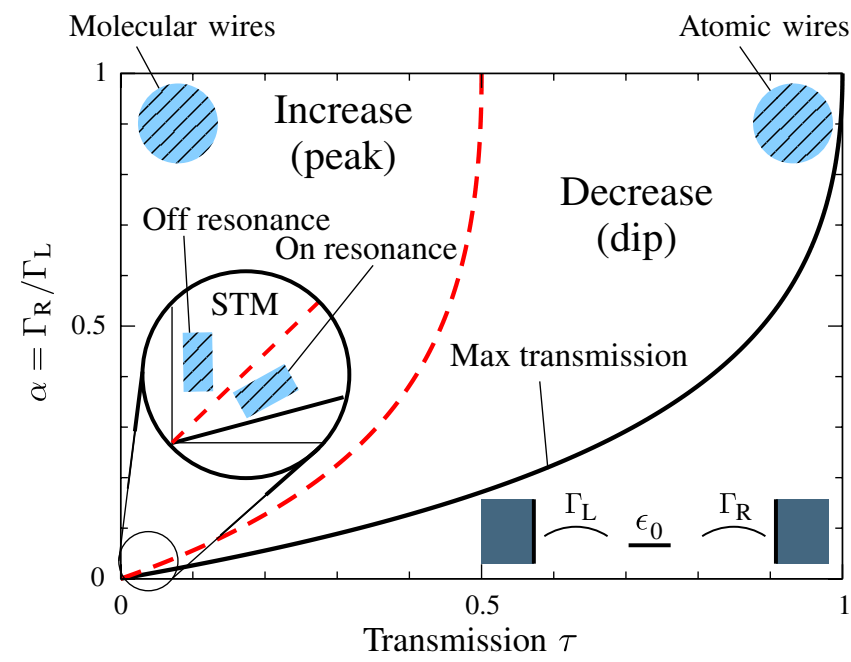

FIG. 1 (color online). Phase diagram for a one-level model (inset) illustrating the sign of the conductance change at the onset of phonon emission. At a given asymmetry factor $\alpha$ the elastic transmission $\tau$ has an upper bound $\tau_{\max }$ (black line), and the inelastic conductance change undergoes a sign change at $\tau_{\text {crossover }}=\tau_{\max } / 2$ (dashed line). A Mathematica notebook for the one-level model is available online [21]. and Gagliardi et al. [14] have extensively discussed the propensity rules.

Instead of trying to understand the complex issues of the competition between elastic and inelastic parts of the conductance, we have found that the phonon emission rate provides a simple way to obtain the IETS propensity rules. In the LOE approximation the power deposited into the phonon system is given by [17]

$$
\begin{aligned}
P^{\mathrm{LOE}}= & \sum_{\lambda} \frac{\left(\hbar \omega_{\lambda}\right)^{2}}{\pi \hbar}\left\{n_{B}\left(\hbar \omega_{\lambda}\right)-n_{\lambda}\right\} \operatorname{Tr}\left[\mathbf{M}_{\lambda} \mathbf{A} \mathbf{M}_{\lambda} \mathbf{A}\right] \\
& +\hbar \omega_{\lambda} \gamma_{\lambda}^{\mathrm{FGR}} \times \begin{cases}0 & ;|e V|<\hbar \omega_{\lambda}, \\
|V|-\frac{\hbar \omega_{\lambda}}{e} & ;|e V|>\hbar \omega_{\lambda} .\end{cases}
\end{aligned}
$$

The first term describes electron-hole damping of the vibrations that drive the actual occupation $n_{\lambda}$ towards the Bose-Einstein equilibrium value $n_{B}$. The second term describes the heating of the phonon system in terms of the emission rate [17]

$$
\gamma_{\lambda}^{\mathrm{FGR}}=\frac{e}{\pi \hbar} \operatorname{Tr}\left[\mathbf{A}_{L} \mathbf{M}_{\lambda} \mathbf{A}_{R} \mathbf{M}_{\lambda}\right]=\frac{4 \pi e}{\hbar} \sum_{l, r}\left|\left\langle\Psi_{l}\left|\mathbf{M}_{\lambda}\right| \Psi_{r}\right\rangle\right|^{2},
$$

where $\mathbf{A}_{L, R}=\mathbf{G} \boldsymbol{\Gamma}_{L, R} \mathbf{G}^{\dagger}$ are the partial spectral functions from the two leads. To provide physical insights we rewrite the trace in terms of a complete set of scattering states $\left|\Psi_{l, r}\right\rangle$ from the left (right) lead. This gives Eq. (4) the form of the physically transparent Fermi's golden rule (FGR). It is advantageous to choose the basis as eigenchannels [20], i.e., the scattering states belonging to the largest transmission. Since the $e$-ph coupling is essentially local in space, it is sufficient to evaluate Eq. (4) using only a few of the most transmitting eigenchannels while the reflected scattering states can be ignored. For the examples described below, only one to three scattering states are needed to account for over $90 \%$ of the phonon scattering.

To illustrate how the phonon emission rate leads to the IETS propensity rules, we have performed calculations on four experimentally realized systems which we believe correspond to the four "corners" of the phase diagram; see Figs. 1 and 2. The calculations were performed within density functional theory (DFT) [25] using our extension of TRANSIESTA as described in Ref. [19]. Broadening by temperature and lock-in modulation $V_{\text {rms }}$ were included.

The IETS, defined as $\left(d^{2} I / d V^{2}\right) /(d I / d V)$, is shown in Fig. 2 for the following:

(a) The symmetric low-transmission case: an oligophenylethylene (OPE) molecule symmetrically thiol-bonded to the hollow position on $\mathrm{Au}(111)$ leads. The temperature and modulation voltage used in the calculation were $T=$ $4.2 \mathrm{~K}$ and $V_{\text {rms }}=8 \mathrm{meV}$. As we have described previously [18], the calculated IETS compare qualitatively with measurements [9].

(b) The symmetric high-transmission case: a 7-atom $\mathrm{Au}$ chain connected to $\mathrm{Au}(100)$ leads, $T=4.2 \mathrm{~K}, V_{\mathrm{rms}}=1 \mathrm{meV}$. There is quantitative agreement with experiments $[4,19]$. 


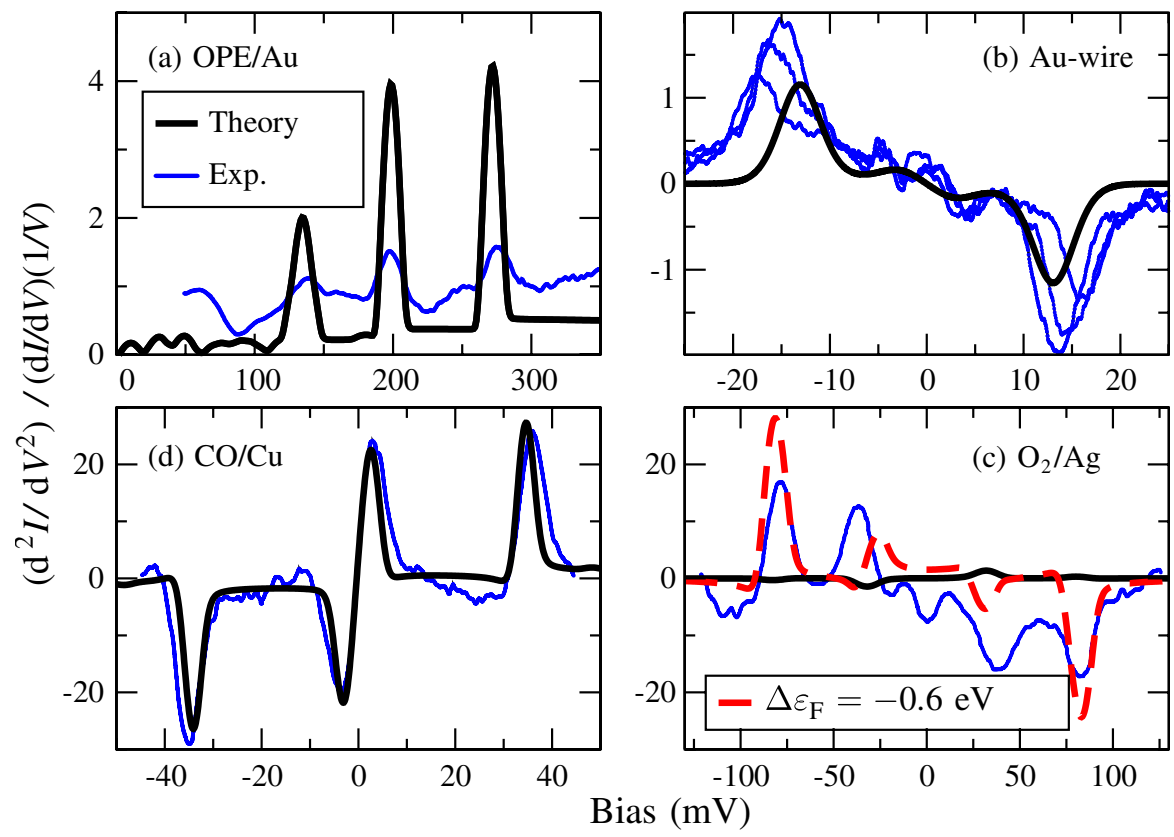

FIG. 2 (color online). Calculated (black lines) and experimental [blue (or gray) lines] IETS representing the corners of the phase diagram in Fig. 1: (a) OPE molecule with $\mathrm{Au}(111)$ leads, (b) Au chain connected to $\mathrm{Au}(100)$ leads, (c) $\mathrm{O}_{2}$ molecule on $\mathrm{Ag}(110)$, and (d) $\mathrm{CO}$ molecule on $\mathrm{Cu}(111)$. In case (c) the Fermi energy $\left(\varepsilon_{\mathrm{F}}\right)$ as been shifted manually to match the experiment (dashed line). The experimental data originate from Refs. [4,9,27,28]. For the STM configurations (c) and (d), the calculated IETS is compared with a rescaled $d^{2} I / d V^{2}$. (c) The on-resonance STM configuration with an $\mathrm{O}_{2}$ molecule on $\operatorname{Ag}(110)$ surface displaying a decrease in the conductance upon phonon emission [27]. The STM tip is modeled by a single $\mathrm{Ag}$ atom on a $\mathrm{Ag}(110)$ lead laterally displaced by $1.6 \AA$ corresponding to the experimental situation [27], $T=13 \mathrm{~K}, V_{\mathrm{rms}}=7 \mathrm{meV}$. The IETS for $\mathrm{O}_{2} / \mathrm{Ag}(110)$ is shown both for the self-consistent DFT calculation (solid black) as well as with $\varepsilon_{\mathrm{F}}$ shifted manually by $-0.6 \mathrm{eV}$ with respect to the DFT result (dashed, low bias conductance $750 \mathrm{nA} / \mathrm{V}$ ).

(d) The off-resonance STM configuration with a CO molecule adsorbed on a $\mathrm{Cu}(111)$ surface [28]. The STM tip is modeled by a single $\mathrm{Cu}$ atom positioned on a $\mathrm{Cu}(111)$ lead, $T=5 \mathrm{~K}, V_{\mathrm{rms}}=2 \mathrm{meV}$.

We find that the size of the conductance drop given by $\gamma^{\mathrm{LOE}}$ is well approximated with the phonon emission rate $\gamma^{\mathrm{FGR}}$. For the OPE and Au-wire cases, the first eigenchannel gives the majority of the phonon emission rate; see Fig. 3. In this case the propensity rules follow from the symmetry of the scattering states which are similar to the Bloch states of the corresponding periodic systems [20]. To illustrate this understanding of the IETS we have published Mathematica notebooks showing the propensity rules for tight-binding models [21]. Before comparing the theoretical STM-IETS to the experimental, we note that the typical tip-sample distances of STM are much larger than what is feasible computationally with a localized basis set. In practice, we work with a significantly smaller tip-sample distances than in the experimental situation, while still being in the tunneling regime.

Results for the $\mathrm{O}_{2}$ on $\mathrm{Ag}(110)$ system are shown in Fig. 2(c). In this case our first-principles calculation fails to describe the conductance decreases observed experimentally [25]. The DFT-based energy spectrum does not have a molecular resonance at $\varepsilon_{\mathrm{F}}$, c.f., the on-resonance case of Fig. 1. However, DFT predicts a $\pi^{*}$ resonance
$0.6 \mathrm{eV}$ below $\varepsilon_{\mathrm{F}}$ [26]. By manually adjusting $\varepsilon_{\mathrm{F}}$ to this resonance we manage to capture some of the qualitative features of the IETS. Although this result is suggestive, we have no conclusive evidence that the experimental decrease is caused by this mechanism.

The results for $\mathrm{CO} / \mathrm{Cu}(111)$ [25] is shown in Figs. 2(d) and 4. The heights of the IETS peaks are qualitatively captured, we predict that $13 \%$ of the transmitted electrons will emit a frustrated rotation phonon compared to $8 \%$ found experimentally for $\mathrm{CO} / \mathrm{Cu}(001)$ [29,30]. The eigenchannels necessary to calculate the transition rates $\gamma^{\text {FGR }}$ are shown in Fig. 4(a). We note that the primary eigenchannel is of $\sigma$ type, i.e., rotationally symmetric around the tip-molecule direction, while the secondary and tertiary are

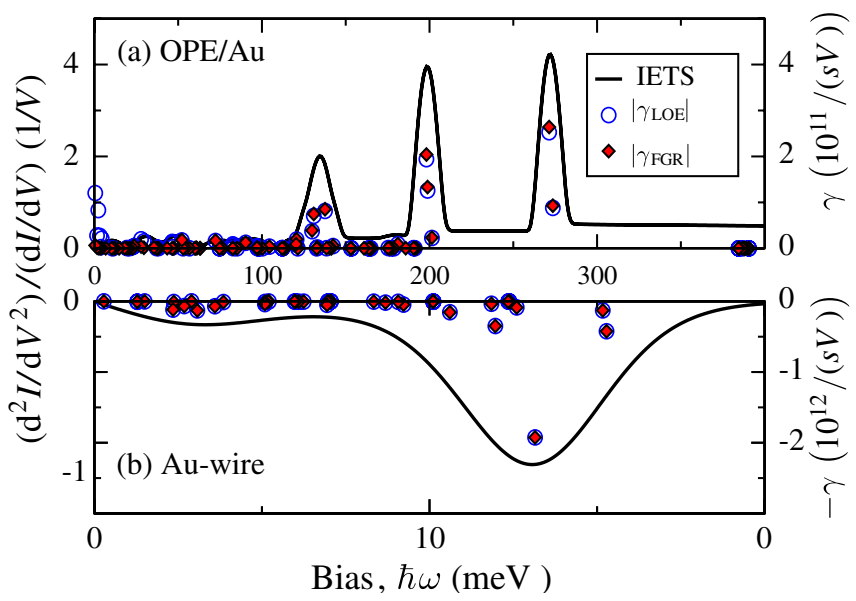

FIG. 3 (color online). Comparison between the FGR scattering rate, calculated using only the single-most transmitting eigenchannel, with the full LOE rate for (a) OPE molecule with $\mathrm{Au}(111)$ leads [compared with the zero-bias conductance of $\left.1.0 \times 10^{13} \mathrm{e} /(\mathrm{s} \mathrm{V})\right]$, and (b) 7 -atom Au wire $\left[4.8 \times 10^{14} \mathrm{e} /(\mathrm{s} \mathrm{V})\right]$. The sign of the FGR rate was chosen to agree with the LOE rate. 
(a)

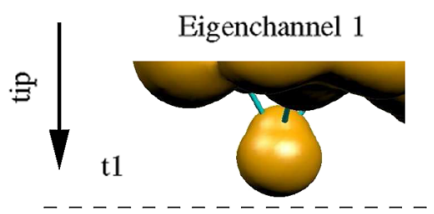

$\mathrm{t} 2$

Eigenchannel 2

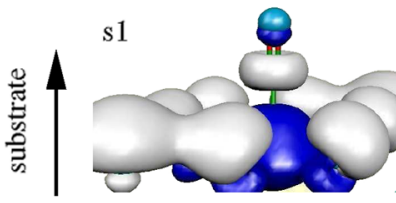

$\mathrm{s} 2$

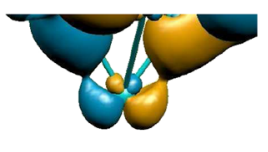

ש

(b)

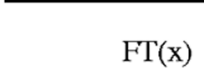
$3 \mathrm{meV}$
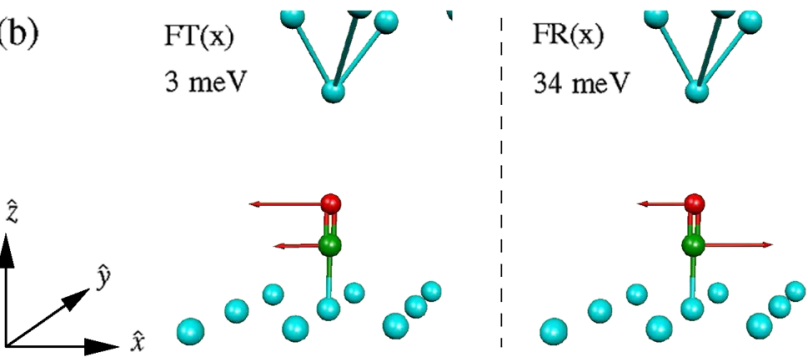

FIG. 4 (color online). Eigenchannels and phonon modes for $\mathrm{CO}$ on $\mathrm{Cu}(111)$. (a) Scattering states associated with eigenchannel 1 (left column, $\tau_{1}=1.9 \times 10^{-3}$ ) and eigenchannel 2 (right column, $\tau_{2}=0.4 \times 10^{-3}$ ). The top (bottom) row of scattering states originates from the tip (substrate) side. Eigenchannel 3 (not shown, $\tau_{3}=0.4 \times 10^{-3}$ ) closely resembles those of eigenchannel 2 rotated by $90^{\circ}$. (b) $\mathrm{FR}(x)$ and $\mathrm{FT}(x)$ vibrational modes [degenerate with $\operatorname{FR}(y)$ and $\operatorname{FT}(y)$ ].

of $\pi$ type. Comparing the scattering states to the phonon modes, Fig. 4(b), the propensity rules naturally follow from the symmetry. The frustrated translation $\left(\mathrm{FT}_{x, y}\right)$ and frustrated rotation $\left(\mathrm{FR}_{x, y}\right)$ modes (in the surface $x y$ plane) can only scatter between a $\pi$ - and a $\sigma$-type eigenchannel, because the modes have $\pi$ character with respect to the transport direction, i.e., $s 2 \leftrightarrow t 1$ and $s 3 \leftrightarrow t 1$. Note that the $s 1 \leftrightarrow t 2,3$ transitions are less important due to the weight of the scattering states (Fig. 4). Similarly, the $\sigma$-type $\mathrm{FT}_{z}$ and $\mathrm{CO}$ stretch modes only scatter between eigenchannels of the same type, e.g., $s 1 \leftrightarrow t 1$. Table I confirms these

TABLE I. Vibrational modes and inelastic scattering rates for $\mathrm{CO}$ on $\mathrm{Cu}(111)$. The FGR rate Eq. (4) (using all eigenchannels) and the LOE rate Eq. (1) are given in units of $10^{10}(\mathrm{~s} \mathrm{~V})^{-1}$ [elastic conductance $\left.=130 \times 10^{10} \mathrm{e} /(\mathrm{s} \mathrm{V})\right]$. The dominating transitions between the eigenchannel scattering states are indicated along with the fraction in which these processes contribute to the total FGR rate.

\begin{tabular}{ccccc}
\hline \hline$\hbar \omega(\mathrm{meV})$ & $\gamma_{\mathrm{FGR}}\left(\gamma_{\mathrm{LOE}}\right)$ & Substrate $\leftrightarrow$ tip & $\%$ & Mode \\
\hline 236 & $0.9(0.8)$ & $s_{i} \leftrightarrow t_{i}, i=1,2,3$ & 100 & CO stretch \\
48 & $0.3(0.3)$ & $s_{i} \leftrightarrow t_{i}, i=2,3$ & 95 & $\operatorname{FT}(z)$ \\
35 & $8.2(8.0)$ & $s 3 \leftrightarrow t 1$ & 95 & $\operatorname{FR}(y)$ \\
34 & $8.3(8.1)$ & $s 2 \leftrightarrow t 1$ & 95 & $\operatorname{FR}(x)$ \\
3 & $5.9(5.8)$ & $s 3 \leftrightarrow t 1$ & 92 & $\operatorname{FT}(y)$ \\
3 & $6.1(6.0)$ & $s 2 \leftrightarrow t 1$ & 92 & $\operatorname{FT}(x)$ \\
\hline \hline
\end{tabular}

observations and gives a quantitative account of the $e$-ph scattering in the $\mathrm{CO} / \mathrm{Cu}(111)$ system. It shows that $\gamma^{\mathrm{FGR}}$ closely approximates the $\gamma^{\mathrm{LOE}}$ and that the main part is given by the first three eigenchannels.

We have presented a method to analyze inelastic $e$-ph scattering in terms of eigenchannel scattering states. The main advance in the context of first-principles transport calculations is to bring the description into a natural framework where underlying symmetries of the propensity rules can be understood. Through four different examples corresponding to different transport regimes, we demonstrated that the eigenchannel analysis addresses the propensity rules in a unified way.

*magnus.paulsson@hik.se

[1] B. C. Stipe, M. A. Rezai, and W. Ho, Science 280, 1732 (1998).

[2] T. Komeda et al., Science 295, 2055 (2002).

[3] J. I. Pascual et al., Nature (London) 423, 525 (2003).

[4] N. Agraït et al., Phys. Rev. Lett. 88, 216803 (2002).

[5] X. Qiu, G. Nazin, and W. Ho, Science 299, 542 (2003).

[6] B. J. LeRoy et al., Nature (London) 432, 371 (2004).

[7] M. Lastapis et al., Science 308, 1000 (2005).

[8] M. Kiguchi et al., Phys. Rev. Lett. 98, 146802 (2007).

[9] J. G. Kushmerick et al., Nano Lett. 4, 639 (2004).

[10] A. Troisi et al., Proc. Natl. Acad. Sci. U.S.A. 104, 14255 (2007).

[11] R. H. M. Smit et al., Nature (London) 419, 906 (2002).

[12] A. Troisi and M. A. Ratner, J. Chem. Phys. 125, 214709 (2006).

[13] N. Lorente et al., Phys. Rev. Lett. 86, 2593 (2001).

[14] A. Gagliardi et al., Phys. Rev. B 75, 174306 (2007).

[15] T. Mii, S. G. Tikhodeev, and H. Ueba, Phys. Rev. B 68, 205406 (2003).

[16] M. Galperin et al., Science 319, 1056 (2008).

[17] M. Paulsson, T. Frederiksen, and M. Brandbyge, Phys. Rev. B 72, 201101(R) (2005).

[18] M. Paulsson, T. Frederiksen, and M. Brandbyge, Nano Lett. 6, 258 (2006).

[19] T. Frederiksen et al., Phys. Rev. B 75, 205413 (2007).

[20] M. Paulsson and M. Brandbyge, Phys. Rev. B 76, 115117 (2007).

[21] http://www.magnuspaulsson.se/FGRpaper.

[22] L. de la Vega et al., Phys. Rev. B 73, 075428 (2006).

[23] O. Tal et al., Phys. Rev. Lett. 100, 196804 (2008).

[24] H. Ueba, T. Mii, and S. Tikhodeev, Surf. Sci. 601, 5220 (2007).

[25] A discussion of the DFT related issues with the position of the $\pi^{*}$ resonance of $\mathrm{O}_{2} / \mathrm{Ag}(111)$ [26] and the $\mathrm{CO}$ adsorption on $\mathrm{Cu}(111)$ are outside the scope of this work.

[26] F. E. Olsson, N. Lorente, and M. Persson, Surf. Sci. 522, L27 (2003).

[27] J. R. Hahn, H. J. Lee, and W. Ho, Phys. Rev. Lett. 85, 1914 (2000).

[28] A. J. Heinrich et al., Science 298, 1381 (2002).

[29] L. J. Lauhon and W. Ho, Phys. Rev. B 60, R8525 (1999).

[30] M. Persson, Phil. Trans. R. Soc. A 362, 1173 (2004). 